\title{
Progesterona plasmática de ovelhas submetidas ao efeito-macho e mantidas sob diferentes condições nutricionais
}

\author{
[Plasmatic progesterone of ewes submitted to male effect and kept at different nutritional conditions] \\ A. Sasa ${ }^{1}$, K.O. Nonaka², J.C.C. Balieiro ${ }^{3}$, L.A. Coelho ${ }^{3}$ \\ ${ }^{1}$ Universidade Estadual de Mato Grosso do Sul \\ Rodovia Aquidauana-UEMS km 12 \\ Aquidauana, MS \\ ${ }^{2}$ Universidade Federal de São Carlos, São Carlos, SP \\ ${ }^{3}$ Universidade de São Paulo, Pirassununga, SP
}

\begin{abstract}
RESUMO
Dosou-se a concentração plasmática de progesterona $\left(\mathrm{P}_{4}\right)$ em ovelhas Santa Inês (SI), Suffolk (SU) e Romney Marsh (RM) em anestro sazonal e submetidas ao efeito-macho, as quais receberam ou não suplementação alimentar. Machos vasectomizados foram introduzidos no grupo de fêmeas após um período prévio de isolamento de 60 dias, e amostras de sangue foram colhidas antes e após a introdução dos machos. Houve efeito $(\mathrm{P}<0,01)$ de período, raça, interação período x raça e interação suplementação x período x raça sobre a concentração de P4. Nas ovelhas SI ocorreu aumento $(\mathrm{P}<0,01)$ da concentração de P4 após a introdução do macho, indicando que houve aumento na atividade cíclica reprodutiva desse grupo. A suplementação, neste caso, potencializou este aumento. Nas ovelhas SU e RM não ocorreram modificações na concentração de $\mathrm{P}_{4}$ $(\mathrm{P}>0,01)$ após a introdução dos machos, nem houve efeito de suplementação. O efeito-macho foi eficaz em induzir a atividade reprodutiva durante o anestro sazonal em ovelhas SI, mas não em ovelhas SU e RM, e a associação dessa prática com a suplementação alimentar é recomendada para ovelhas da raça nativa SI.
\end{abstract}

Palavras-chave: ovino, anestro, atividade reprodutiva

\begin{abstract}
The present study aimed to verify the plasmatic progesterone $\left(\mathrm{P}_{4}\right)$ concentrations in Santa Inês (SI), Suffolk (SU) and Romney Marsh (RM) ewes submitted to male effect receiving or not food supplementation during seasonal anestrous. Vasectomized rams were introduced to the group of ewes after 60 day isolation. Blood samples were collected before and after the male's introduction. Effects $(P<0.01)$ of period, breed, period $x$ breed interaction and period $x$ breed $x$ supplementation interaction on plasmatic $P_{4}$ concentrations were observed. The plasmatic $P_{4}$ concentrations of SI ewes increased $(P<0.01)$ after the male introduction, indicating there was an increase in the reproductive activity in this group. Supplementation increased this effect. For $\mathrm{SU}$ and $\mathrm{RM}$ ewes the plasmatic $\mathrm{P}_{4}$ concentrations did not alter $(\mathrm{P}>0.01)$ after the male introduction, with no supplementation. It was concluded the male effect was efficient in inducing the reproductive activity in SI ewes, but not in SU and RM ewes. The association of the effect of the male with supplementation increased this effect.
\end{abstract}

Keywords: anestrous, ovine, reproductive activity

\section{INTRODUÇÃO}

A maioria das ovelhas apresenta um modelo de reprodução sazonal, sendo o fotoperíodo um dos principais fatores responsáveis por essa estacionalidade. As ovelhas iniciam a estação reprodutiva à medida que a luminosidade diária diminui, obedecendo ao fotoperíodo decrescente, ou seja, a incidência de ciclos estrais está concentrada durante o outono e o inverno (Jainundeen et al., 2004). A resposta da atividade sexual ao fotoperíodo é, também, dependente da latitude. Em latitudes mais elevadas, a estacionalidade reprodutiva está intimamente

Recebido em 10 de setembro de 2010

Aceito em 14 de abril de 2011

E-mail: aya@uems.br 
relacionada com o fotoperíodo, enquanto em baixas latitudes essa relação é menos pronunciada.

A estacionalidade da atividade cíclica reprodutiva das fêmeas afeta o sistema de produção de ovinos de corte por restringir a produção de cordeiros para abate anual. Dessa forma, reduzir a duração do período de anestro e antecipar a estação reprodutiva constituem importantes fatores econômicos a serem considerados na ovinocultura de corte. Um método prático para alcançar esses objetivos é o condicionamento das fêmeas em anestro ao isolamento, seguido pela introdução dos machos. Esse método é conhecido como efeito-macho e sua eficiência pode variar conforme a região, a época do ano, o grau de estacionalidade reprodutiva e o peso corporal das fêmeas (Nugent III et al., 1988; Otto et al, 1998; Skinner et al., 2002; Véliz et al., 2006).

Outra forma de incrementar a reprodução das ovelhas é mediante o uso do manejo nutricional conhecido como flushing (Gunn et al., 1991; Kott, 2001), que consiste no aumento da oferta de energia aos animais, antes e durante a estação reprodutiva (Neary, 2001). Entretanto, existem poucas informações sobre a suplementação alimentar associada ao efeito-macho.

A determinação da concentração plasmática de progesterona tem sido utilizada como um método de monitoramento da atividade cíclica reprodutiva em ovelhas (Minton, 1990; Guerin et al., 2000; Zarazaga et al., 2003). Fêmeas são consideradas em período anovulatório (anestro) quando a concentração plasmática permanece abaixo de $1,0 \mathrm{ng} / \mathrm{mL}$ em mais de duas amostras consecutivas de sangue ou por um período igual ou acima de 10 dias (Minton et al., 1991). Outros pesquisadores consideram o período de atividade ovulatória quando duas ou mais amostras consecutivas mostram valores plasmáticos entre 0,4 e $1,0 \mathrm{ng} / \mathrm{mL}$ (Guerin et al., 2000; Zarazaga et al., 2003).

Assim, este trabalho teve o objetivo de avaliar a concentração plasmática de progesterona em ovelhas das raças Santa Inês, Romney Marsh e Suffolk, em época de anestro sazonal, antes e após serem submetidas ao efeito-macho, as quais receberam ou não suplementação alimentar.

\section{MATERIAL E MÉTODOS}

O experimento foi realizado na cidade de Pirassununga-SP, durante época de anestro sazonal - setembro a novembro. A cidade está localizada a $21^{\circ} 59$ de latitude sul, $47^{\circ} 26$ de longitude oeste de Greenwich e 634m de altitude. O clima é classificado como subtropical (Köppen, 1948), a precipitação pluviométrica anual é de $1300 \mathrm{~mm}$ e a temperatura média de $21^{\circ} \mathrm{C}$, sendo a mínima de $13^{\circ} \mathrm{C}$ e a máxima de $35^{\circ} \mathrm{C}$.

Foram utilizadas 36 ovelhas adultas das raças Santa Inês $(n=10)$, Suffolk $(n=15)$ e Romney Marsh ( $\mathrm{n}=11)$ e três machos vasectomizados. As fêmeas foram previamente isoladas - visual e olfativamente - dos machos por 60 dias, após o que estes foram introduzidos no grupo, e aí permaneceram por 40 dias. Todos os animais, mantidos em sistema de pastejo (Panicum maximum cv. Aruana) durante o dia, e confinados no período noturno, em condições de fotoperíodo natural, receberam sal mineral e água à vontade. Metade das fêmeas de cada grupo recebeu suplementação com concentrado comercial, 21 dias antes da introdução e durante a permanência dos machos, a outra metade não recebeu suplementação. O tratamento com suplementação forneceu de 100 a 110\% dos requisitos das necessidades proteicas $(\mathrm{PB})$ e de 130 a $140 \%$ dos requisitos energéticos (NDT), enquanto 0 tratamento não suplementado forneceu de 60 a $70 \%$ de PB e $100 \%$ de NDT, segundo o NRC (Nutrient..., 1985).

Para dosar a concentração plasmática de progesterona, amostras de sangue foram colhidas da veia jugular das fêmeas em dois períodos: antes (período 1) e após (período 2) à introdução do macho no grupo genético. No período 1 , as colheitas foram realizadas durante 21 dias, e no período 2 durante 40 dias, a cada dois ou três dias. Todas as amostras de sangue foram centrifugadas, e o plasma obtido estocado a $-20^{\circ}$ C até o momento da análise.

A concentração plasmática de progesterona foi analisada, em duplicatas, pelo método de radioimunoensaio (RIE) em fase sólida. Para isso, foram utilizados conjuntos de reagentes comerciais (COAT-A-COUNT - Diagnostic Products Company, Los Angeles, CA, USA), 
desenvolvidos para avaliação quantitativa dos hormônios, sem qualquer tipo de extração química e processo de purificação, valendo-se do iodo $^{125}\left(\mathrm{I}^{125}\right)$ como elemento radioativo traçador. Os procedimentos utilizados foram os especificados pelo fabricante. A contagem de radioatividade foi obtida pela utilização de contador gama PACKARD, modelo 5000, calibrado automaticamente para $\mathrm{I}^{125}$. As análises foram realizadas em dois ensaios, sendo o limite de detecção de $0,02 \mathrm{ng} / \mathrm{mL}$, e os coeficientes de variação inter e intraensaios foram de $2,5 \%$ e de $1,6 \%$, respectivamente.

O monitoramento da atividade cíclica reprodutiva foi realizado por meio da determinação da concentração plasmática de progesterona e da observação de estros usando machos equipados com buçal marcador. A dosagem da concentração de $\mathrm{P}_{4}$ antes da introdução do macho teve o objetivo de identificar as fêmeas que estavam em atividade cíclica reprodutiva e as que se encontravam em anestro sazonal. De acordo com Minton (1990), valores plasmáticos de progesterona abaixo de $1,0 \mathrm{ng} / \mathrm{mL}$ caracterizam a fase. A fase de anestro diferencia-se da fase de estro quando a concentração de progesterona permanece baixa por um período maior que 10 dias. Para avaliar o retorno da atividade cíclica reprodutiva, foram considerados somente os animais que se encontravam em anestro - valores plasmáticos de $\mathrm{P}_{4}$ abaixo de 1,0ng/mL por 10 ou mais dias - antes da introdução do macho. A partir da presença dos machos, ovelhas que apresentaram aumento da concentração plasmática de $\mathrm{P}_{4}-$ acima de 2,0ng/mL - foram consideradas em atividade (retorno) cíclica reprodutiva.

Os dados da concentração plasmática de progesterona foram avaliados mediante análise de variância, utilizando-se o procedimento PROC MIXED do SAS (Statistical..., 1995), com opção REPEATED, seguido pelo teste Tukey para estabelecer a comparação entre médias.
Foram considerados os efeitos principais de tratamento - suplementação e não suplementação -, período - antes e após introdução dos machos -, e raça - Santa Inês, Suffolk e Romney Marsh - e o efeito das interações duplas e tripla entre os fatores.

\section{RESULTADOS E DISCUSSÃO}

Observaram-se efeitos $(\mathrm{P}<0,01)$ de raça, período, interação raça $x$ período e interação raça $x$ período $\mathrm{x}$ tratamento sobre a concentração plasmática de progesterona. As maiores concentrações foram observadas $(\mathrm{P}<0,01)$ nas fêmeas Santa Inês $(2,21 \pm 0,13 \mathrm{ng} / \mathrm{mL})$, seguidas das fêmeas Suffolk $(0,47 \pm 0,04 \mathrm{ng} / \mathrm{mL})$ e Romney Marsh $(0,24 \pm 0,01 \mathrm{ng} / \mathrm{mL})$. De maneira geral, a concentração foi mais baixa $(\mathrm{P}<0,01)$ antes da introdução do macho (Fig. 1) em relação à não introdução $-0,58 \pm 0,06$ versus $0,98 \pm 0,06 \mathrm{ng} / \mathrm{mL}$.

Quanto ao efeito da interação raça x período, a maior $(\mathrm{P}<0,01)$ concentração da progesterona plasmática ocorreu nas fêmeas Santa Inês após a introdução dos machos - 2,52 $\pm 0,16 \mathrm{ng} / \mathrm{mL}$-, seguidas pelas mesmas fêmeas antes da

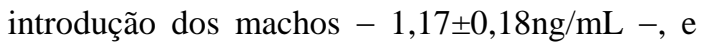
por último, concentrações abaixo de 1,00ng/mL nas fêmeas Romney Marsh e Suffolk, nos dois períodos.

A interação tripla raça $\mathrm{x}$ período $\mathrm{x}$ tratamento mostra que as concentrações mais altas ocorreram nas fêmeas suplementadas da raça Santa Inês após a introdução dos machos 2,32 $\pm 0,22 \mathrm{ng} / \mathrm{mL}-$, seguidas pelas fêmeas não suplementadas da mesma raça antes da introdução do macho - 1,65 $\pm 0,22 \mathrm{ng} / \mathrm{mL}$. As menores concentrações - abaixo de $1,0 \mathrm{ng} / \mathrm{mL}$ foram observadas nas fêmeas Suffolk e Romney Marsh. 

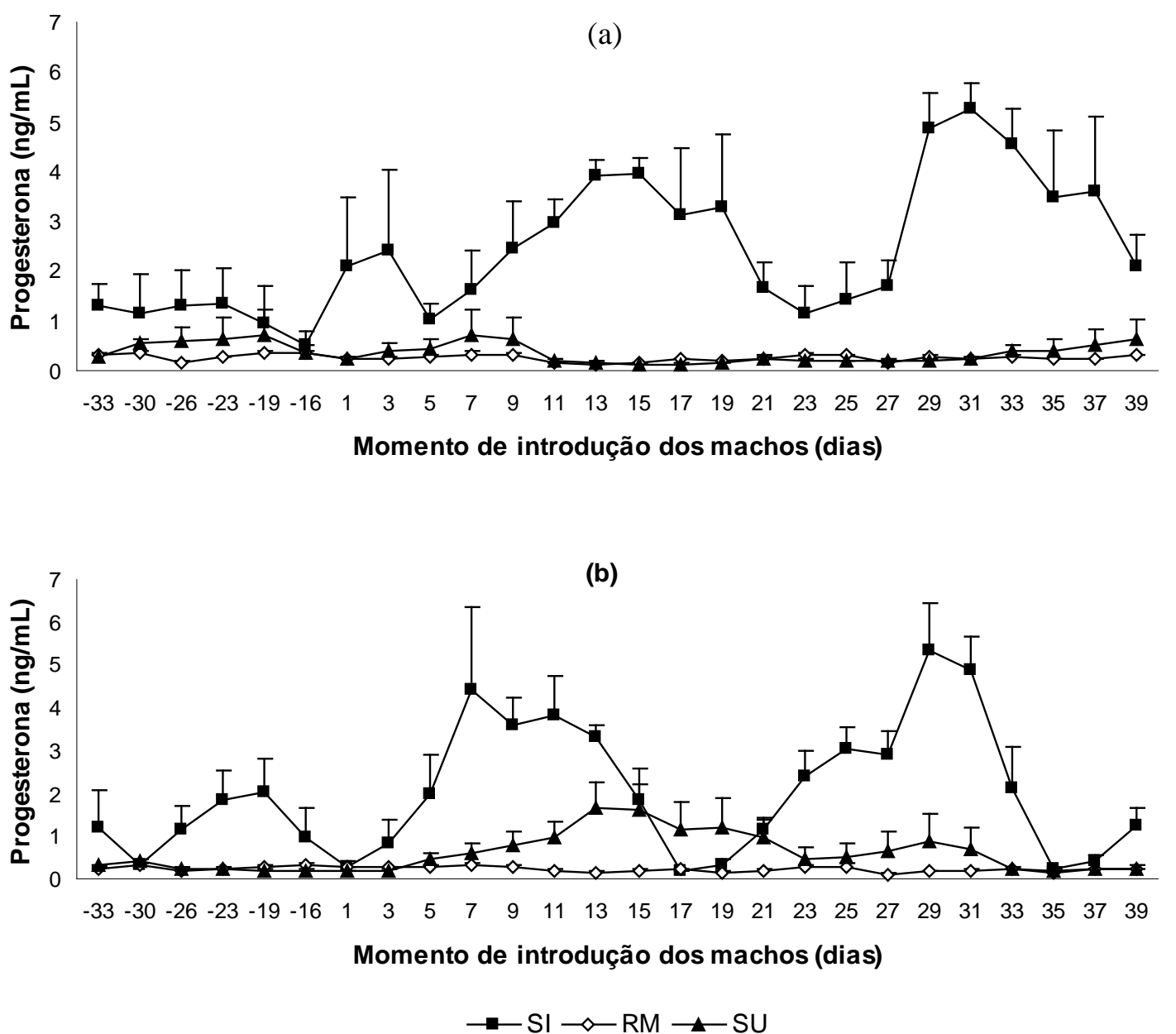

Figura 1. Médias e erros-padrão da concentração plasmática de progesterona (ng/mL) em ovelhas das raças Santa Inês (SI), Romney Marsh (RM) e Suffolk (SU) em anestro sazonal, antes e após a introdução dos machos, e que receberam (a) ou não (b) suplementação alimentar.

O perfil plasmático individual de progesterona observado na raça Santa Inês mostrou que três das cinco fêmeas do grupo suplementado e duas das cinco fêmeas do grupo não suplementado encontravam-se em atividade cíclica reprodutiva antes da introdução do macho. Após a introdução, todas as fêmeas, inclusive as que estavam em anestro, apresentaram concentração de progesterona maior que $1,0 \mathrm{ng} / \mathrm{mL}$. Nas fêmeas dessa raça, a concentração média sempre se manteve acima de $1,0 \mathrm{ng} / \mathrm{mL}$. Isso indica que, em geral, elas encontravam-se em atividade cíclica reprodutiva antes da introdução do macho, e que, após sua introdução, intensificouse a atividade cíclica reprodutiva. A suplementação neste grupo teve efeito positivo sobre a concentrações da progesterona plasmática. No grupo suplementado, houve um aumento de 1,09 para 2,32ng/mL após a introdução do macho, enquanto no grupo não suplementado esse aumento foi menos pronunciado - de 1,26 para 1,65ng/mL.

Para as fêmeas Suffolk, houve ligeiro aumento da concentração plasmática de progesterona após a introdução do macho, reflexo da atividade ovulatória de apenas cinco fêmeas. Antes da introdução, somente uma fêmea do grupo suplementado e nenhuma do grupo não suplementado apresentavam atividade cíclica. Após a introdução, a mesma fêmea que estava em atividade cíclica reprodutiva e mais quatro do 
grupo não suplementado encontravam-se em atividade cíclica. Nestas, o efeito-macho foi pouco evidente, e a suplementação não exerceu efeito.

Nas fêmeas Romney Marsh, o efeito-macho não foi efetivo. Todas se encontravam em anestro e mantiveram essa condição após a introdução do macho. Isso refletiu na concentração de progesterona, que permaneceu abaixo de $1,00 \mathrm{ng} / \mathrm{mL}$ ao longo de todo o período, tanto nas suplementadas como nas não suplementadas.

Embora não seja possível afirmar que existam diferenças entre raças quanto à atividade reprodutiva em razão do número limitado de animais avaliados por grupo racial, o resultado obtido confirma os verificados em pesquisas realizadas anteriormente, em condições subtropicais no Sudeste do Brasil, as quais mostraram diferentes graus de estacionalidade reprodutiva em fêmeas Santa Inês, Romney Marsh e Suffolk (Coelho et al., 2006; Sasa, 2006; Rodrigues et al., 2007). Esses autores sugeriram que as raças Santa Inês, Suffolk e Romney Marsh podem ser classificadas com baixo, intermediário e alto grau de estacionalidade reprodutiva, respectivamente.

Assim, este trabalho mostrou que a resposta ao efeito-macho pode variar em razão do grau de estacionalidade reprodutiva das fêmeas, e que a condição nutricional colabora para tal, ou seja, o efeito-macho pode ser potencializado se associado à correta condição alimentar. Estes resultados confirmam os obtidos em outras pesquisas realizadas em regiões temperadas (Skinner et al., 2002). Em animais com baixo grau de estacionalidade reprodutiva, a simples introdução do macho é capaz de estimular o retorno da atividade cíclica ovariana, porém, em fêmeas com alto grau de estacionalidade reprodutiva, o efeito-macho não tem sido capaz de ativar a atividade reprodutiva durante a época de anestro sazonal. De acordo com Gordon (1997), quanto maior a sensibilidade ao fotoperíodo nas raças originárias de regiões temperadas, maior é o período de anestro, e menor será a resposta ao estímulo como efeitomacho. Flores et al. (2000) sugeriram que a não resposta ao efeito-macho no período de anestro existe muito mais em razão do reduzido estímulo do macho do que à falta de resposta da fêmea. Para esses autores, o macho também diminui a atividade sexual na época de anestro das fêmeas. Para tornar efetivo o efeito-macho durante o anestro, seria necessário submeter o macho (Delgadillo et al., 2002) ou as fêmeas (PellicerRubio et al., 2007) a um prévio tratamento fotoperiódico associado ou não à administração de melatonina (Delgadillo et al., 2001).

No presente estudo, a introdução do macho no grupo de fêmeas consideradas com baixo grau de estacionalidade reprodutiva foi suficiente para que as que estavam em anestro retornassem à atividade reprodutiva. Por outro lado, a resposta ao efeito-macho na raça Suffolk, considerada com grau intermediário de estacionalidade reprodutiva (Sasa et al., 2001), foi baixa - 4/15 diferente dos resultados obtidos por Otto et al. (1998), que trabalharam com fêmeas dessa raça na região Sul do Brasil e obtiveram uma resposta parcial a esse efeito. Essa discrepância pode ser atribuída ao pequeno número de ovelhas avaliadas no presente estudo e às condições nutricionais oferecidas, isto é, apenas pastejo de Panicum maximum cv. Aruana. Essa condição alimentar - grupo não suplementado - não atendeu totalmente às exigências nutricionais das fêmeas em reprodução, ao fornecer apenas 60 a $70 \%$ das exigências em proteína bruta e 100\% das de energia bruta segundo o NRC (Nutrient..., 1985). De acordo com Hulet et al. (1986), as condições nutricionais também podem interferir no percentual de ovelhas em anestro que respondem ao efeito macho. Ovelhas que apresentam baixa condição nutricional tendem a permanecer em anestro, não respondendo ao efeito-macho.

As fêmeas Romney Marsh, que apresentam alto grau de estacionalidade reprodutiva, não modificaram o perfil plasmático de progesterona em razão do efeito-macho e da suplementação. Rosa et al. (2000), ao trabalharem com ovelhas de alto grau de estacionalidade reprodutiva da raça Northcountry Mule, também não detectaram aumento da porcentagem de ovelhas que ovularam na época de anestro em resposta ao efeito-macho.

No presente experimento, a condição nutricional exerceu efeito sobre a concentração de progesterona somente nas fêmeas Santa Inês. Rondina et al. (2005) verificaram que o incremento na condição nutricional em cabras proporcionou aumento dos níveis da progesterona 
plasmática e, consequentemente, da atividade estral.

Segundo Martin et al. (2004), raças de origem temperada, como a Suffolk, apresentam estacionalidade reprodutiva dirigida pelo fotoperíodo, enquanto raças tropicais, como a Merino, apresentam sazonalidade reprodutiva mais em razão da disponibilidade de alimentos. E estas últimas podem ter o ciclo reprodutivo anual facilmente modificado por interação sociossexual. Isto, provavelmente, justifica o que foi observado nas fêmeas da raça Santa Inês.

\section{CONCLUSÕES}

Em ovelhas com baixo grau de estacionalidade reprodutiva, como a raça nativa Santa Inês, o efeito-macho proporciona aumento na concentração plasmática de progesterona e retorno mais rápido da atividade cíclica reprodutiva. Quando esta prática está associada à suplementação alimentar, o incremento da progesterona plasmática é mais acentuado. Em ovelhas com intermediário e alto grau de estacionalidade reprodutiva, como as raças Suffolk e Romney Marsh, o efeito macho, mesmo associado à suplementação alimentar, não é capaz de promover aumento na concentração plasmática de progesterona nem de anular a condição de anestro.

\section{REFERÊNCIAS BIBLIOGRÁFICAS}

COELHO, L.A.; RODRIGUES, P.A.; NONAKA, K.O. et al. Annual pattern of plasma melatonin and progesterone concentrations in hair and wool ewe lambs kept under natural photoperiod at lower latitudes in the southern hemisphere. J. Pin. Res., v.41, p.101-107, 2006.

DELGADILLO, J.A.; FLORES, J.A.; VÉLIZ, F.G. et al. Induction of sexual activity in lactating anovulatory female goats using male goats treated only with artificially long days. $J$. Anim. Sci., v.80, p.2780-2786, 2002.

DELGADILLO, J.A.; CARRILLO, E.; MORÁN, J. et al. Induction of sexual activity of male creole goats in subtropical northern México using long days and melatonin. J. Anim. Sci., v.79, p. 2245-2252, 2001.
FLORES, J.A.; VÉLIZ, F.G.; PÉREZVILLANUEVA, J.A. et al. Male reproductive condition is the limiting factor of efficiency in the male effect during seasonal anestrus in female goats. Biol. Reprod., v.62, p.1409-1414, 2000.

GORDON, I. Controlled reproduction in sheep and goats. Cambridge: CABI Publishing, 1997. v.2, 450p.

GUERIN, M.V.; DEED, J.R.; MATTHEWS, C.D. The coincidence of light and melatonin with a specific phase of the circadian pacemaker is important for the timing of seasonal breeding in the ewe. J. Biol. Rhythms, v.15, p.514-523, 2000.

GUNN, R.G.; MAXWELL, T.J.; SIM, D.A. et al. The effect of level of nutrition prior to mating on the reproductive performance of ewes of two welsh breeds in different levels of body condition. Anim. Prod., v.52, p.157-163, 1991.

HULET, C.V.; SHUPE, W.L.; ROSS, T. et al. Effects of nutritional environment and ram effect on breeding season in range sheep. Theriogenology, v.25, p.317-323, 1986.

JAINUDEEN, M.R.; WAHID, H.; HAFEZ, E.S.E. Ovinos e caprinos. In: HAFEZ, E.S.E.; HAFEZ, B. Reprodução animal. 7.ed. São Paulo: Manole, 2004. Cap.12, p.173-182.

KÖPPEN, W. Climatologia. Buenos Aires: Panamericana, 1948. 478p.

KOTT, R. Sheep nutrition. 2001. Disponível em: $<$ http://agadsrv.msu.edu/Extension/Sheep/Handb ook/nutr.html>. Acessado em: 30 jan. 2001.

MARTIN, G.B.; RODGER, J.; BLACHE, D. Nutritional and environmental effects on reproduction in small ruminants. Reprod. Fertil. Dev., v.16, p.491-501, 2004.

MINTON, J.E.; COPPINGER, T.R.; SPAETH, C.W. et al. Poor reproductive response of anestrous Suffolk ewes to ram exposure is not due to failure to secrete luteinizing hormone acutely. J. Anim. Sci., v.69, p.3314-3320, 1991.

MINTON, J.E. Role of photorefractoriness in onset of anoestrus in Ramboullet X Dorset ewes. J. Reprod. Fertil., v.89, p.261-268, 1990.

NUTRIENT requirements of domestic animals: nutrient requirements of sheep. 6.ed. Washington: DC. National Academy, 1985. 99p. 
NEARY, M. Reproductive management of the ewe flock and the ram [200-]. Disponível em: $<$ http://ag.ansc.purdue.edu/sheep/articles/reprom gt.html>. Acessado em: 30 jan. 2001.

NUGENT III, R.A.; NOTTER, D.R.; BEAL, W.E. Effects of ewe breed and ram exposure on estrous behavior in May and June. J. Anim. Sci., v.66, p.1363-1370, 1988.

OTTO, C.; ANDRIGUETTO, J.L.; SÁ, J.L. et al. Estudo do "efeito macho" na concentração dos partos de ovelhas e borregas expostas à monta no anestro sazonal. In: REUNIÃO ANUAL DA SOCIEDADE BRASILEIRA DE ZOOTECNIA, 35., 1998, Botucatu, SP. Anais... Botucatu, SP: SBZ, 1998. p.163-165.

PELLICER-RUBIO, M.T.; LEBOUEUF， B.; BERNELAS, D. et al. Highly synchronous and fertile reproductive activity induced by the male effect during deep anoestrus in lactating goats subjected to treatment with artificially long days followed by a natural photoperiod. Anim. Reprod. Sci., v.98, p.241-258, 2007.

RODRIGUES, P.A.; COELHO, L.A.; NONAKA, K.O. et al. Annual characteristics of estrous activity in wool and hair ewe lambs under subtropical conditions. Sci. Agr., v.64, n.5, 2007.

RONDINA, D.; FREITAS, V.J.F.; SPINACI, M. et al. Effect of nutrition on plasma progesterone levels, metabolic parameters and small follicles development in unstimulated goats reared under Constant photoperiod regimen. Reprod. Dom. Anim., v.40, p.548-552, 2005.

ROSA, H.J.D.; JUNIPER, D.T.; BRYANT, M.J. The effect of exposure to oestrus ewes on rams sexual behaviour, plasma testosterone concentration and ability to stimulate ovulation in seasonally anoestrus ewes. Appl. Anim. Behav. Sci., v.67, p.293-305, 2000.
STATISTICAL analysis system. SAS: user's guide (release 8.02). Cary, NC: SAS Institute, 1995.

SASA, A. Perfis sazonais das concentrações plasmáticas de progesterona, prolactina $e$ melatonina de ovelhas criadas em baixas latitudes. 2006. 50f. Tese (Doutorado em Zootecnia) - Faculdade de Zootecnia e Engenharia de Alimentos, Universidade de São Paulo, Pirassununga, SP.

SASA, A.; TESTON, D.C.; CRIVELLENTI, T.L. et al. Incidência sazonal de estros em borregas lanadas e deslanadas criadas no Estado de São Paulo. In: REUNIÃO ANUAL DA SOCIEDADE BRASILEIRA DE ZOOTECNIA, 38., 2001, Piracicaba, SP. Anais... Piracicaba, SP: SBZ, 2001. p.383-384.

SKINNER, D.C.; CILLIERS, S.D.; SKINNER, J.D. Effect of ram introduction on the oestrus cycle of springbok ewes (Antidorcas marsupialis). Reproduction, v.124, p.509-513, 2002.

VÉLIZ, F.G.; POINDRON, P.; MALPAUX, B. et al. Positive correlation between the body weight of anestrous goats and their response to the male effect with sexually active bucks. Reprod. Nutr. Dev., v. 46, p.657-661, 2006.

ZARAZAGA, L.A.; MALPAUX, B.; CHEMINEAU, P. Amplitude of the plasma melatonin nycthemeral rhythms is not associated with the dates of onset and offset of the seasonal ovulatory activity in the Ile de France ewe. Reprod. Nutr. Dev., v.43, p.167-177, 2003. 